\title{
Implementation of transmission NIR as a PAT tool for monitoring drug transformation during HME processing
}

\author{
Muhammad T. Islam ${ }^{\mathrm{a}}$, Nikolaos Scoutaris ${ }^{\mathrm{a}}$, Mohammed Maniruzzaman ${ }^{\mathrm{a}}$, Hiren G. \\ Moradiya $^{\mathrm{a}}$, Sheelagh A. Halsey ${ }^{\mathrm{b}}$, Michael Bradley ${ }^{\mathrm{a}}$, Babur Z. Chowdhry ${ }^{\mathrm{a}}$, Martin J. \\ Snowden $^{\mathrm{a}}$, Dennis Douroumis ${ }^{\mathrm{a}^{*}}$
}

${ }^{a}$ Faculty of Engineering and Science, University of Greenwich, Medway Campus, Chatham Maritime, Kent ME4 4TB, UK

${ }^{b}$ ThermoFisher Scientific, Stafford House, Boundary Way, Hemel Hempstead HP2 7GE, UK

Abbreviations: API, active pharmaceutical ingredients; DSC, differential scanning calorimetry; FDA, Food and Drug Administration; HME, hot-melt extrusion; IND, indomethacin; NIR, near infrared spectroscopy; PAT, process analytical technology; PCA, principal component analysis; QbD, Quality by design; SOL, soluplus; VA64, Kollidon VA 64; XRPD, x-ray powder diffraction.

\footnotetext{
* Corresponding author, Department of Pharmaceutical, Chemical \& Environmental Sciences, Faculty of Engineering and Science, University of Greenwich, Medway Campus, Chatham Maritime, Kent ME4 4TB, UK. Tel.: +44 (0) 208331 8440; Fax: +44 (0) 2083319805.

E-mail address: D.Douroumis@gre.ac.uk (D. Douroumis).
} 


\begin{abstract}
The aim of the work reported herein was to implement process analytical technology (PAT) tools during hot melt extrusion (HME) in order to obtain a better understanding of the relationship between HME processing parameters and the extruded formulations. For the first time two in-line NIR probes (transmission and reflectance) have been coupled with HME to monitor the extrusion of the water insoluble drug indomethacin (IND) in the presence of Soluplus (SOL) or Kollidon VA64 hydrophilic polymers. In-line extrusion monitoring of sheets, produced via a specially designed die, was conducted at various drug/polymer ratios and processing parameters. Characterization of the extruded transparent sheets was also undertaken by using DSC, XRPD and Raman mapping. Analysis of the experimental findings revealed the production of molecular solutions where IND is homogeneously blended (ascertained by Raman mapping) in the polymer matrices, as it acts as a plasticizer for both hydrophilic polymers. PCA analysis of the recorded NIR signals showed that the screw speed used in HME affects the recorded spectra but not the homogeneity of the embedded drug in the polymer sheets. The IND/VA64 and IND/SOL extruded sheets displayed rapid dissolution rates with $80 \%$ and $30 \%$ of the IND being released, respectively within the first $20 \mathrm{~min}$.
\end{abstract}

Keywords:

Indomethacin

In-line monitoring

Reflectance

Transmission

Near infrared spectroscopy

Process analytical technology

Hot-melt extrusion

Molecular solutions

Raman mapping 


\section{Introduction}

Hot melt extrusion (HME) is an emerging processing technology for the development of solid drug dispersions embedded in a carrier matrix for the development of oral solid dosage pharmaceutical forms [1-5]. The technique has been adopted in pharmaceutical research due to its versatility and the several advantages that it offers compared to traditional manufacturing techniques. For example, HME is solvent free, a wide range of excipients can be processed, it is cost effective, and can be easily scaled-up with high throughput and operated in continuous mode [6]. HME has been utilized to enhance the dissolution rate of APIs by preparing solid dispersions [7, 8] where the drug is molecularly dispersed in the polymer matrix which leads to improved bioavailability [9]. Repka et al, utilized HME to prepare bioadhesive films with increased dissolution rates of poorly water-soluble drugs by exploring the influence of processing parameters [10-12]. The developed films were used for pharmaceutical and denture applications using transmucosal delivery. Sun et al. prepared a semi-solid capsule containing a solid dispersion of nimodipine processed with Eudragit ${ }^{\circledR}$ E100 and Plasdone ${ }^{\circledR}$ S630 using a twin-screw extruder [13]. The extruded solid dispersion was formulated into gelatine capsules followed by in vivo studies in beagle dogs and compared with a marketed product of the same drug. The in vivo comparison revealed faster absorption of nimodipine for the hot-melt extruded formulations compared to the marketed product.

The Food and Drug Administration (FDA) introduced the concept of Process Analytical Technologies (PAT) in 2004 for a better fundamental scientific understanding of manufacturing processes $[14,15]$ and to ensure the final quality of the end product. The aim of this initiative is to implement appropriate measurement tools, which can be placed at/in or on-line for monitoring and data analysis via a scientific approach such as chemometrics and Quality by Design (QbD) approach to ensure production of in-process materials and the final quality of products [16].

HME is among the technologies that can be coupled with PAT tools for the development of pharmaceutical solid dosage forms. In-line reflectance NIR and in-line Raman spectroscopy have also been used as PAT tools in order to understand drug-polymer interactions and to validate a method for continuous API quantification during HME processing [17-18]. The application of in-line NIR spectroscopy to monitor the extrusion process was demonstrated by Fischer et al [19] who successfully determined the content of ethylene vinyl acetate in a polypropylene matrix. Off-line and in-line reflectance NIR has 
also been used to monitor the formation of carbamazepine-saccharine [20] and carbamazepine-trans-cinnamic acid [21] cocrystals.. However, reflectance NIR contains large amount of noise in the spectra when monitoring a transparent formulation. In contrast, transmission NIR has also been reported for studies of single screw polymer extrusion of low [22] and high [23] density polyethylene/polypropylene co-polymers. The use of transmission NIR for the characterization of an API extrusion processed via HME has not been reported. Using NIR as a non-destructive on-line monitoring tool for the impact of critical process parameters (feed-rate, screw speed, temperature) improves process efficiency and product quality as adjustments to the process can be made in real time when deviations in the process are detected during HME. This was shown by Coates et al [23] for polyethylene-propylene blends where rapid and accurate determination of the ethylene content is essential. Thus it is important to employ an analytical method, such as transmission NIR, for monitoring transparent extrudates of an API produced by HME [17].

In this study, a transmission NIR probe was used for in-line monitoring of transparent extruded sheet formulations; the NIR probe was inserted in a specially designed extrusion die. In addition HME processing was coupled with both transmission and reflectance NIR probes in order to provide a more detailed understanding of the physical transformations of the drug during extrusion. For the purposes of the study, indomethacin (IND) was extruded with the hydrophilic polymers Soluplus (SOL) or Kollidon VA64 (VA64) at various drug/polymer ratios. Previous studies showed that the extrusion of IND with these polymers leads to the production of molecular solutions with improved drug dissolution rates compared to the bulk drug [9].

\section{Materials and methods}

\subsection{Materials}

Indomethacin (IND) was supplied by Tokyo Chemical Industry Co. Ltd (Tokyo, Japan), Soluplus (polyvinyl caprolactam-polyvinyl acetate-polyvinyl-glycol graft copolymer) and Kollidon VA64 (vinylpyrrolidone-vinyl acetate copolymer) were kindly donated by BASF (Ludwigshafen, Germany). HPLC solvents (acetonitrile, acetic acid) were of analytical grade and purchased from Fisher Scientific (Loughborough, UK). All chemicals were used as received. 


\subsection{Hot-melt extrusion process}

HME was performed by using a Eurolab $16 \mathrm{~mm}$ twin screw extruder (Thermo Fisher Scientific, Karlsruhe, Germany). The HME extruder was equipped with a DD Flexwall@ 18 volumetric feeder (Brabender Technology, Duisburg, Germany). Seven different formulations of IND/SOL and IND/VA64, at different weight ratios, were extruded (Table 1). Prior to HME, drug-polymer batches $(300 \mathrm{~g})$ were blended in a Turbula TF2 mixer (100 rpm) (Basel, Switzerland) for $10 \mathrm{~min}$ in order to obtain a homogeneous mix. The barrel temperature profile was set at 50/100/120/135/145/150/150/150/140 ${ }^{\circ} \mathrm{C}$ (from feeder to die) for batches F1-F5 and at 50/100/120/150/150/150/150/150/140 ${ }^{\circ} \mathrm{C}$ for formulations $\mathrm{F} 6$ and F7. A newly manufactured transmission die (Fig. 1A.) was fitted at the end of the extruder barrel in order to collect in-line transmission NIR spectra (Fig. 2). The die was made of stainless steel, with a $1 \mathrm{~mm}$ die opening where the transmission NIR probe can be inserted and the distance between the incident and the transmitted radiation is therefore $1 \mathrm{~mm}$ (slit size) so that the infra-red radiation can pass through the transparent extrudates and be transmitted to the NIR detector.

The extruded sheets were fed continuously to a cutter mill (Cutting Mill SM 100, Retsch, Uk) for further micronization with constant rotating speed. A $250 \mu \mathrm{M}$ sieve was placed at the bottom of the casing holds to control the size of the material discharged from the milling zone.

\subsection{Thermal analysis}

The physical state of bulk IND, the physical mixtures and the extruded granules was analysed by DSC using a Mettler-Toledo 823e (Greifensee, Switzerland) instrument. Accurately weighed samples (2-3 mg) were placed in aluminium pans and sealed. Measurements were carried out under an atmosphere of dry nitrogen gas at a flow rate of $50 \mathrm{~mL} / \mathrm{min}$ using a heating rate of $10.0^{\circ} \mathrm{C} / \mathrm{min}$ from $0^{\circ} \mathrm{C}$ to $180^{\circ} \mathrm{C}$.

Drug-polymer miscibility is related to the shift(s) in the melting endotherm or glass transition temperature of the drug [32]. Based on the Gordon-Taylor equation [24], if the drug and polymer are miscible then the mixture will show a single $T \mathrm{~g}$ that ranges between the $T \mathrm{~g}$ of the pure components and would depend on the relative proportion of each component. The $\mathrm{Tg}$ of the mixtures can be predicted using the Gordon-Taylor (GT) equation:

$$
\operatorname{Tg} \operatorname{mix}=\frac{w 1 \times \operatorname{Tg} 1+K \times \mathrm{w} 2 \times \operatorname{Tg} 2}{w 1+K \times \mathrm{w} 2}
$$


Where, $T \mathrm{~g}$ is the glass transition temperature, $w 1$ and $w 2$ are the weight fractions of components and $K$ is calculated from the densities $(\rho)$ and $T \mathrm{~g}$ of the amorphous components:

$$
K=\frac{\operatorname{Tg} 1 \times \rho 1}{\operatorname{Tg} 2 \times \rho 2}
$$

\subsection{X-ray powder diffraction}

XRPD was used to assess the crystalline state of the IND, the physical mixtures and the hot-melt extrudates. All formulations including pure IND, physical mixtures and extruded formulations were evaluated using a Bruker D8 (Karlsruhe, Germany) Advance instrument in theta-theta mode, using a $\mathrm{Cu}$ anode at $40 \mathrm{kV}$ and $40 \mathrm{~mA}$, parallel beam Goebel mirror, 0.2 $\mathrm{mm}$ exit slit, LynxEye Position Sensitive Detector with $3^{\circ}$ opening and LynxIris at $6.5 \mathrm{~mm}$ and sample rotation at $15 \mathrm{rpm}$. The samples were scanned from 2 to $40^{\circ} 2$-theta with a step size of $0.02^{\circ} 2$-theta and a counting time of $0.2 \mathrm{~s}$ per step; 176 channels active on the PSD resulting in a total counting time of 35.2 s per step.

\subsection{Near-infrared spectroscopy (NIR)}

Transmission and reflectance NIR spectra were continuously collected in-line and noninvasively during hot-melt extrusion using a Fourier-Transform Antaris MX NIR analyser (Thermo Fisher Scientific, United Kingdom) equipped with a InGaAs detector, a quartz halogen lamp, and a fibre optic probe which was attached in the extrusion die. Spectra were collected every $1 \mathrm{~min}$ in the $10000-4000 \mathrm{~cm}^{-1}$ wavenumber region with a resolution of $16 \mathrm{~cm}^{-1}$ and averaged over 32 scans. For each batch 20 spectra were recorded. A transmission probe was fitted at the end of the extruder orifice (die) and the reflectance probe was inserted in the first mixing zone of the extruder barrel (Fig. 2).

NIR data analysis was performed by using RESULT Software (Version 3.0, Thermo Fisher Scientific, UK). Spectra collected in both diffuse reflectance and transmission modes usually require spectral pre-treatment before analysis. The degree of scattering depends on the wavelength of the incident radiation and the refractive index of the sample, which causes an uneven scatter over the whole spectrum. This can result in a baseline shift. Therefore, standard normal variate (SNV) correction was used before chemometric analysis of the spectra. Using SNV, unwanted scatter was removed from the raw spectra to prevent it from dominating over chemical information within the spectra. The result of SNV pre-processing was that each spectrum had the same offset and amplitude, eliminating differences in light scatter in the spectra from different samples, before developing the calibration model. 
Furthermore, second derivative pre-processing was undertaken after SNV correction. Second derivative NIR spectra magnify differences in spectral features, provide baseline normalisation and remove linear data offsets due to scattering effects and path length variation. For principal component analysis (PCA) and for the development of a partial least square (PLS) model, 10 spectra of each drug-polymer mixture were used. Prior to PCA and PLS, spectra were mean centred. The PLS model was developed by regressing the IND concentration $(Y)$ versus the corresponding in-line collected spectra $(X)$. The validated spectra were used to evaluate the predictive performance of the PLS model.

\subsection{Confocal Raman spectroscopy}

Raman mapping was performed using a Jobin/Yvon LabRam 320 instrument equipped with an Olympus microscope (Horiba, Japan) by means of a He-Ne ion laser $(\lambda=632.8 \mathrm{~nm})$ and $1800 \mathrm{l} / \mathrm{nm}$. The experimental conditions were: $100 \mathrm{~nm}$ slit width, a $50 \times$ Microsoft objective and $0.2 \mathrm{~s}$ acquisition times. Each spectrum was the mean of two. The sample profiling was performed at step increments of $8 \mu \mathrm{m}$ in the $X-Y$ direction over an area of $2000 \times 600 \mu^{2}$. PCA was used for data analysis. Prior to the analysis, all spectra were base line corrected and normalised using the standard normal variate method (SNV) to avoid intensity deviation among the Raman spectra. The Raman chemical maps were constructed by using Solo + Mia software (Eigenvector, Research, Inc. Wenatchee, USA).

\subsection{In-vitro drug release studies}

The release profiles for IND (bulk and granules produced from the extruded sheets using a cutter mill) were obtained using a Varian 705 DS dissolution paddle apparatus (Varian Inc. North Carolina, US). The analysis was carried out using dosage forms containing $50 \mathrm{mg}$ of IND, in $900 \mathrm{~mL}$ of dissolution medium maintained at $37.0 \pm 0.5^{\circ} \mathrm{C}$ and agitated at $100 \mathrm{rpm}$. A phosphate buffer solution $\mathrm{pH} 6.8$ was used the dissolution medium. At pre-established time intervals $5 \mathrm{~mL}$ samples were withdrawn from the dissolution medium, filtered through a $0.45 \mu \mathrm{m}$ mesh, and analysed using HPLC. All dissolution studies were performed in triplicate.

\subsection{HPLC analysis}

The release of IND, in the dissolution studies, was determined by using an Agilent Technologies HPLC 1200 series system equipped with a HICROM S50DS2, $5 \mu \mathrm{m}$ x $150 \mathrm{~mm}$ x $4 \mathrm{~mm}$ column using a $20 \mu \mathrm{l}$ injection volume and a detection wavelength of $298 \mathrm{~nm}$. The 
mobile phase consisted of acetonitrile/water $(1 \%$ acetic acid) $(70: 30 \mathrm{v} / \mathrm{v})$. The flow rate was adjusted to $1.5 \mathrm{~mL} / \mathrm{min}$ and the retention time of IND was about $4 \mathrm{~min}$. A calibration curve for IND (10-50 $\mu \mathrm{g} / \mathrm{mL})$ was used to quantitate all the samples.

\section{Results and discussion}

\subsection{Hot-melt extrusion process}

The objective of this study was to investigate, for the first time, the implementation of in-line transmission and reflectance NIR probes in order to understand the transformation of the API (IND), in the presence of the polymers, from a crystalline phase to an amorphous phase during HME processing. The use of an NIR transmission probe for in-line monitoring during HME has not been previously reported and in this case it was used to record the NIR signals from molecular solutions or amorphous solid dispersions. It is known that a drugpolymer system is considered miscible if the difference between their solubility parameters is less than 7.0 $\mathrm{MPa}^{1 / 2}[25]$. Binary blends with differences in solubility parameters higher than $10 \mathrm{MPa}^{1 / 2}$ are likely to be immiscible. Maniruzzaman et al [10] showed that the solubility parameters for IND, VA64 and SOL are $22.84 \mathrm{MPa}^{1 / 2}, 19.63 \mathrm{MPa}^{1 / 2}$ and $19.40 \mathrm{MPa}^{1 / 2}$, respectively. The selection of the drug/polymer formulations was expected to show good compatibility as the solubility parameter differences between IND/VA64 and INS/SOL are $3.21 \mathrm{MPa}^{1 / 2}$ and $3.44 \mathrm{MPa}^{1 / 2}$ respectively. Hence, glassy solutions were obtained during HME processing due to the excellent miscibility of IND with both polymers. Using a die orifice (Fig. 1A) with a $1 \mathrm{~mm}$ slit size, results in the production of IND/polymer extrudates in the form of transparent sheets (Fig. 1B). The sheet thickness was also $1 \mathrm{~mm}$, which facilitated the monitoring process using the in-line NIR transmission probe. Formulations F1-F5 were extruded at various drug-polymer ratios using a constant, low screw speed (50 rpm) in order to gain a better understanding of the transformations in the crystallinity of the API. However, higher screw speeds were applied for formulations F6 and F7 in order to monitor NIR signal variations related to the screw speed. Heil et al., [26] observed that higher screw speeds result in greater variability in extrusion processing, whereas lower screw speeds provide better repeatability.

\subsection{X-ray powder diffraction (XRPD)}

XRPD analysis was performed in order to investigate the physical state of the bulk IND, drug-polymer physical mixtures and the hot-melt extruded samples. The data in Fig. 3A shows the diffractogram of bulk IND with distinct intensity peaks at $10.17^{\circ}, 11.62^{\circ}, 17.02^{\circ}$, 
$19.60^{\circ}, 21.82^{\circ}, 23.99^{\circ}, 26.61^{\circ}, 29.37^{\circ}, 30.32^{\circ}$ and $33.55^{\circ} 2 \theta$ values whereas pure SOL and VA64 presented noisy signals due to their amorphous nature. The physical mixtures of IND formulations with both SOL and VA64 polymers showed lower intensity peaks suggesting that they remained in the crystalline state at 20-50\% loading (Fig. S1). In contrast, no distinct intensity peaks were observed in the diffractograms of all the extruded formulations, even at high drug loading (Fig. 3B). The absence of high intensity IND peaks indicates the formation of a solid dispersion, where the drug is present in an amorphous state or molecularly dispersed into the polymer matrices.

\subsection{Differential scanning calorimetry}

Differential scanning calorimetry was also used to determine the physical state of IND within the extruded formulations and compared with those of the physical mixtures. As shown in the data in Fig. 4A the DSC thermograms of pure IND displayed sharp melting endothermic transitions at $161.08^{\circ} \mathrm{C}\left(\Delta \mathrm{H}=101.60 \mathrm{Jg}^{-1}\right)$. The DSC thermograms of SOL and VA64 gave $T \mathrm{~g}$ values of $68.07^{\circ} \mathrm{C}$ and $104.99^{\circ} \mathrm{C}$, respectively due to their amorphous nature (Fig. 4A). Interestingly, the thermograms of the physical mixtures of all IND/VA64 and IND/SOL formulations showed broad endothermic transitions (Fig. 4B-C). The absence of a sharp melting peak for IND in the physical mixtures suggests that the API is partially dissolved within the polymer matrices. This behaviour is attributed to the both SOL and VA64 acting as solubilizers for a wide range of water insoluble drugs [27].

However, a single $T \mathrm{~g}$ was observed in all thermograms for the extrudates indicating drug-polymer miscibility. According to the GT equation, when two components of a mixture are miscible the single $T \mathrm{~g}$ of the extruded materials should be located between their $\mathrm{Tg}$ values [33]. By using the Gordon-Taylor equation the theoretical $T \mathrm{~g}$ of the drug-polymer blends was estimated (Table 2). In addition, the presence of a single $T \mathrm{~g}$ in all extruded samples suggests that the IND is molecularly dispersed within the polymer matrices. The incorporation of IND in both polymers lowers the $T \mathrm{~g}$ and allows the polymer chain segments greater freedom due to the fact that IND acts as a plasticizer. In addition, the comparison of the experimental Tg's and those obtained by the GT equation revealed that IND acts as a plasticizer for SOL as the Tgs were found in - between the drug and the polymer. It can be seen that the Tg decreases with the increase of IND concentrations. Similar results were obtained for the IND/VA64 extudates which are in good agreement with previous studies [10,33]. 


\subsection{In-line NIR monitoring}

Off-line NIR spectra of bulk IND, SOL and VA64 and the physical mixtures of all formulations were measured in order to identify the characteristic bands attributed to the pure samples. Using both the in-line NIR transmission and reflectance probes the HME process was monitored for all of the IND formulations. The probes were placed in two different positions of the extruder barrel; with the reflectance NIR probe fitted into the first mixing zone of the barrel and the transmission NIR probes placed in the extruder exit die (Fig. 2). The rationale for using both probes was to provide a more detailed understanding of the drug transformations during extrusion. As the first mixing zone was near to the feed point, it was expected that the processed formulations would not have been fully melted in this particular zone of the extruder; and a significant IND amount remained in crystalline form. This allowed the spectra to be collected using the reflectance NIR probe in this zone as the mixture would still be reflective. On the other hand, the extrudates were converted to a transparent solution along the mixing zones 2-3 so the spectra were collected using a transmission NIR probe placed at the extruder die.

Two additional formulations (F6 and F7) were extruded in order to identify the effect of screw speed on the extrusion process; screw speeds of $200 \mathrm{rpm}$ and $400 \mathrm{rpm}$ were applied during the IND/SOL (50/50, w/w) extrusion. Principal component analysis (PCA) was used to investigate whether or not NIR could be used to detect the results of changes in the screw speed of the extruder. PCA was applied over the $6500-5500 \mathrm{~cm}^{-1}$ spectral range to 50 spectra (10 spectra each from IND/SOL formulations, F3-F7) resulting in a model with two PCs covering nearly all the spectral variations (99.04\%). The PC scores plot obtained using constant process parameters (F3 - F5) and variable screw speeds (F6 and F7) are shown in Fig. 5. The PC scores plot clearly shows that the screw speed has a significant effect on the NIR spectra as samples were widely spread on the PC1 axis. This effect is attributed to the changes in the die pressure and torque when the screw speed is increased. For thixotropic and pseudoplastic polymers there is usually a liner relationship between the screw speed and torque or die pressure. Table 3 shows the monitoring of the torque and die pressure as a function of screw speed. It can be clearly seen that by increasing the screw speed from 50 $\mathrm{rpm}$ to $400 \mathrm{rpm}$ the torque increases from $8.8 \mathrm{Nm}$ to $12.7 \mathrm{Nm}$, respectively. Similarly, the die pressure increases with the screw speed and thus affects the NIR recorded spectra. The second PC was then used to describe the variation in the IND concentration. Ellipses were drawn around points in the PC score plot corresponding to specific production runs at 
constant IND concentration. However, the ellipses were very similar in size, which indicated that screw speed had very little influence on the homogeneity of the extrudates. PCs model variance and the maximum variance is modelled in PC1, which usually explains more variation than PC2. This means that pressure (screw speed) has a bigger effect on the spectra than IND concentration. That is why extruder conditions have to be kept constant when using an NIR method as changes in processing conditions will have a large influence on the results.

Two binary IND/SOL mixtures containing 20\% and 40\% IND respectively, were extruded under the same extrusion settings $\left(50 \mathrm{rpm}\right.$ screw speed, $\left.145^{\circ} \mathrm{C}\right)$ to investigate the effect of drug concentration in the in - line NIR spectra. Fig. 6a shows the reflectance spectra of the bulk IND, the physical mixture, the processed mixture $\left(Z 1,2^{\text {nd }}\right.$ barrel) and the transmission spectra of the IND/VA64 (20\% and 40\% loading) at the extrusion die. The spectral comparison of the IND/VA64 (40/60 w/w) clearly shows peak shifts, which suggest molecular interactions between the drug and the polymer. The shift is smaller for the reflectance probe $\left(5243 \mathrm{~cm}^{-1}\right)$ in the first mixing zone $\left(100^{\circ} \mathrm{C}\right)$ compared to the physical mixture $\left(5217 \mathrm{~cm}^{-1}\right)$ as IND is still crystalline and not fully transformed to amorphous. The use of the transmission probe in the third mixing zone showed further peak shifts $\left(5251 \mathrm{~cm}^{-1}\right)$ where IND was molecularly dispersed within the polymer matrix. In addition, the increase of IND concentration resulted in larger shifts and higher intensity peaks. The increase of the intensity demonstrates the plasticization effect of IND, which leads to the formation of stronger, and more drug-polymer intermolecular bonds. Similar results can be observed in Fig. $6 \mathrm{~b}$ where the increase of IND concentration in the IND/SOL mixtures presented significant peak shifts with increased peak intensities. Hence, the IND/SOL $(50: 50 \mathrm{w} / \mathrm{w})$ extrudates showed the highest peak intensity $\left(4690 \mathrm{~cm}^{-1}\right)$ compared to the $40 / 60 \mathrm{w} / \mathrm{w}(4682$ $\left.\mathrm{cm}^{-1}\right)$ and 20/80 wt/wt $\left(4664 \mathrm{~cm}^{-1}\right)$ extrudates respectively.

Furthermore, Fig. S2 shows a new peak with high intensity was found at $7100 \mathrm{~cm}^{-1}$ in the all IND/SOL extrudates, which is attributed to the- $\mathrm{OH}$ first overtone stretch. The peak does not appear in the physical mixtures and it is probably due to the H-bonding between the $\mathrm{COOH}$ of IND and the $-\mathrm{OH}$ of SOL. The polymer has an $-\mathrm{OH}$ absorption peak at $7034 \mathrm{~cm}^{-1}$, which shifts to $7100 \mathrm{~cm}^{-1}$ due to the intermolecular interactions of the drug/polymer functional groups.

In Fig. S3(a,b) the NIR spectrum of IND shows a well-defined and intense peak at $6020 \mathrm{~cm}^{-1}$ and similar peaks appear across the whole spectrum. However, all extruded IND/SOL 
formulations showed quite broad and less defined peaks at the third mixing zone for the spectra recorded with the transmission probe. According to previous studies [17, 23] the presence of broad peaks suggests that IND is transformed to an amorphous form and the molecule is not present in a crystalline long-range three-dimensional order. The peak intensity and position of the spectra of the physical mixture shows no drug - polymer interactions which suggests that the drug transformation was effectively achieved during the HME processing.

The NIR data are in good agreement with the DSC thermograms and XRPD diffractograms obtained for the extrudates. The use of the transmission NIR probe was proved helpful in monitoring the HME processing of molecularly dispersed IND/SOL and IND/VA64 that are in the form of clear and transparent extrudates. The use of the reflectance NIR probe for in-line monitoring of such extrudates would be very difficult, because of the high noise signals produced.

\subsection{Confocal Raman spectroscopy}

The data in Fig. 7 illustrates the Raman spectra of the pure compounds. Most of the Raman bands overlap or can be potentially overlapped in the extrudates due to the chemical environment. Hence, for example, the Raman bands of IND in the 1500-1720 $\mathrm{cm}^{-1}$ wavenumber region, which correspond to $\mathrm{C}=\mathrm{O}$ stretching, are overlapped with the broad bands of VA64 at 1650 and $1750 \mathrm{~cm}^{-1}$. Therefore, multivariate data analysis (namely PCA) which takes into account the whole spectrum was applied. The method does not require the pure spectra of the compounds which can change in the formulations due to molecular interactions. In PCA, the spectra are decomposed into principal components that reflect variations amongst the spectra. In principle, the Raman hyperspectral data set can be described as a linear combination of a small number of components and hence instead of observing the distribution of a particular band, the distribution of the entire spectrum which corresponds to a particular component is obtained.

The data in Fig. 8 shows the first principal component spectrum and the corresponding concentration map for the extruded IND/VA64 sample. The hyperspectral Raman data were decomposed into three PCs. There are various methods which can be used to determine the number of components present in PCA [31, 32]. The most common approach is the analysis of eigenvalues and by monitoring the values of the associated scores and loadings. It is quite likely that there are only two components present (Fig. 9a); which is 
expected as there are only two compounds present. This can be clearly confirmed by plotting the first three PCs where only the first PC has identifiable bands. The $2^{\text {nd }}$ and $3^{\text {rd }}$ PCs contain noise and the bands are too broad to extract any useful information (Fig. 8b). The first loading spectrum contains characteristic bands for both IND and VA64. It is worth mentioning that the carbonyl band of IND at $1710 \mathrm{~cm}^{-1}$ moves towards a lower wavenumber (Fig. 8c) indicating stronger interactions of the $\mathrm{C}=\mathrm{O}$ group. It is possible for the carbonyl groups of IND to interact with the $\mathrm{OH}$ groups of other IND molecules via hydrogen bonding. In the amorphous state these interactions are stronger than in crystalline powder resulting in the movement of the $\mathrm{C}=\mathrm{O}$ band to lower wavenumbers. Also, previous studies showed that the Raman bands of amorphous IND become broader [31] resulting in an overlap with the bands of VA64 rendering the analysis even more difficult (Fig. 8b). The concentration map for PC1 (Fig. 8d) shows the homogeneous distribution of the compounds.

\subsection{In-vitro drug release studies}

In-vitro drug release studies were performed in order to investigate the dissolution patterns of the extruded samples and the bulk IND. The particle size of the extruded granules did not have an effect on the dissolution profiles. The extruded sheets were passed through a cutter mill and all batches presented similar particle size distribution (Fig. S4). The dissolution profiles of bulk IND and extruded formulations with the highest IND loadings for VA64 and SOL are shown in Fig. 9. Due to low water solubility, the bulk IND powder showed very low dissolution rates compared with extruded formulations. IND/VA64 extrudates at 40:60 (w/w) ratios displayed a rapid dissolution rate, with $80 \%$ of the IND being released in $15 \mathrm{~min}$ while complete IND release was detected at $30 \mathrm{~min}$. For the extruded IND/SOL granules $30 \%$ of the IND was released in the first $20 \mathrm{~min}$ and approximately $55 \%$ after $30 \mathrm{~min}$. More than $80 \%$ IND was released after $90 \mathrm{~min}$ from the IND/SOL $(50: 50 \mathrm{w} / \mathrm{w})$ extrudates. The dissolution studies (Fig. S5) revealed faster IND release rates with increasing loadings for both polymers due to stronger drug/polymer interactions [33]. However, dissolution studies performed at $\mathrm{pH} 1.2$ showed negligible amount of IND release after $2 \mathrm{hr}$, the findings are in agreement with the previous studies [27].

\section{Conclusions}

In this study, in-line transmission and reflectance NIR probes were employed as PAT tools for monitoring the HME processing of IND/ SOL and IND/VA 64 extrudates. By using both the NIR probes it was possible to monitor the transformation of IND from the crystalline 
to molecularly dispersed state during the extrusion processing. DSC and XRPD analysis of the extruded IND/VA64 and IND/SOL sheets confirmed the plasticizing effect of IND and the manufacture of solid solutions with IND being molecularly dispersed in the polymer matrices. The PCA plots obtained from the NIR transmission spectra showed that a change in the screw speed of the extruder resulted in different NIR spectra due to the increased pressure at the die. The transmission probe also helped to identify stronger drug-polymer interactions with increasing drug concentrations, the formation of $\mathrm{H}$-bonding between their functional groups and IND transformation to a molecular solution within the polymer matrix. Confocal Raman spectroscopic data was used to show the homogeneity of the IND/polymer extruded sheets. In conclusion, the use of a transmission NIR probe proved to be a valuable PAT tool for monitoring and process understanding of HME solid dispersions.

\section{Conflict of interest}

There is no conflict of interest.

\section{Appendix A. Supplementary material}

Supplementary data associated with this article can be found, in the online version.

\section{References}

1. M. Maniruzzaman, J.S. Boateng, M.J. Snowden, D. Douroumis, A review of hot-melt extrusion: process technology to pharmaceutical products, ISRN. 2012. DOI: $10.5402 / 2012 / 436763$.

2. D. Douroumis, Hot-melt extrusion: pharmaceutical applications, First ed., John Wiley \& Sons Ltd. Oxford, UK, (2012).

3. G.P. Andrews, D.S. Jones, Hot melt extrusion- processing solid solutions? J. Pharm. Pharmacol. 66 (2) (2014) 145-147.

4. M. Maniruzzaman, D.J. Morgan, A.P. Mendham, J. Pang, M.J. Snowden, D. Douroumis, Drug-polymer intermolecular interactions in hot-melt extruded solid dispersions, Int. J. Pharm. 43 (1-2) (2013) 199-208.

5. M.M. Crowley, F. Zhang, M.A. Repka, S. Thumma, S.B. Upadhye, S.K. Battu, J.W. McGinity, C. Martin, Pharmaceutical applications of hot-melt extrusion: part I, Drug Dev. Ind. Pharm. 33 (9) (2007) 909-926.

6. A. Almeida, B. Claeys, J.P. Remon, C. Vervaet, Hot-melt extrusion developments in the pharmaceutical industry, In: Douroumis D, (ed.) Hot-melt extrusion: pharmaceutical application, John Wiley \& Sons Ltd, Oxford, UK, (2012) 43-69. 
7. D.S. Jones, D.N. Margetson, M.S. McAllister, T. Yu, L. Shu, C.P. McCoy, G.P. Andrews, Thermodynamically stable amorphous drug dispersions in amorphous hydrophilic polymers engineered by hot melt exttusion, Chem. Eng. Res. Des. (2014) DOI: 10.1016/j.cherd.2014.08.022.

8. S. Qi, P. Belton, K. Nollenberger, N. Clayden, M. Reading, D.Q.M. Craig, Characterisation and prediction of phase separation in hot-melt extruded solid dispersions: A thermal, microscopic and NMR relaxometry study, Pharm. Res. 27 (2010) 1869-1883.

9. M. Maniruzzaman, M.M. Rana, J.S. Boateng, J.C. Mitchell, D. Douroumis, Dissolution enhancement of poorly water soluble APIs processed by hot melt extrusion using hydrophilic polymers, Drug Dev. Ind. Pharm. 39 (2) (2013) 218-227.

10. J.B. Park, S. Prodduturi, J. Morott, V.I. Kulkarni, M.R. Jacob, S.I. Khan, S.P. Stodghill, M.A. Repka, Development of an antifungal denture adhesive film for oral candidiasis utilizing hot melt extrusion technology, Expert Opin. Drug Deliv. 12 (1) (2015) 1-13.

11. M. Chen, J. Lu, W. Deng, A. Singh, N.N. Mohammed, M.A. Repka, C. Wu, Influence of processing parameters and formulation factors on the bioadhesive, temperature stability and drug release properties of hot-melt extruded films containing miconazole, AAPS PharmSciTech. 15 (3) (2014) 522-529.

12. S.M. Trey, D.A. Wicks, P.K. Mididoddi, M.A. Repka, Delivery of itraconazole from extruded HPC films, Drug Dev. Ind. Pharm. 33 (7) (2007) 727-735.

13. Y. Sun., Y. Rui, Z. Wenliang, X. Tang, Nimodipine semi-solid capsules containing solid dispersion for improving dissolution, Int. J. Pharm. 359 (1-2) (2008) 144 -149

14. T. De Beer, A. Burggraeve, M. Fonteyne, L. Saerens, J.P. Remon, C. Vervaet, Near infrared and Raman spectroscopy for the in-process monitoring of pharmaceutical production processes, Int. J. Pharm. 417 (2011) 32- 47.

15. L. Saerens, C. Vervaet, J.P. Remon, T. De Beer, Process monitoring and visualization solutions for hot-melt extrusion: a review, J. Pharm. Pharmacol. 66 (2) (2014) 180203.

16. Food and Drug Administration, http://www.fda.gov/downloads/Drugs/GuidanceComplianceRegulatoryInformation/G uidances/ucm070305.pdf. (2004.)

17. L. Saerens, L. Dierickx, T. Quinten, P. Adriaensens, R. Carleer, C. Vervaet, J.P. Remon, T. De Beer, In-line NIR spectroscopy for the understanding of polymer-drug 
interaction during pharmaceutical hot-melt extrusion. Eur. J. Pharm. Biopharm. 81 (2012) 230-237.

18. V.S. Tumuluri, M.S. Kemper, I.R. Lewis, S. Prodduturi, S. Majumdar, B.A. Avery, M.A. Repka, Off-line and on-line measurements of drug-loaded hot-melt extruded films using Raman spectroscopy, Int. J. Pharm. 357 (1-2) (2008) 77-84.

19. D. Fischer, T. Bayer, K.J. Echhorn, M. Otto, In-line process monitoring on polymer melts by NIR spectroscopy, J. Anal. Chem. 359 (1997) 74-77.

20. H. Moradiya, M.T. Islam, G.R. Woollam, I.J. Slipper, S. Halsey, M.J. Snowden, D. Douroumis, Continuous cocrystallization for dissolution rate optimization of a poorly water-soluble drug, Cryst. Growth Des. 14 (2014) 189-198.

21. H. Moradiya, M.T. Islam, S. Halsey, M. Maniruzzaman, B. Chowdhry, M.J. Snowden, D. Douroumis, Continuous cocrystallisation of Carbamazepine and transCinnamic acid via melt extrusion processing, CrystEngComm. 16 (2014) 3573-3583.

22. T. Rohe, W. Becker, S. Kolle, N. Eisenreich, P. Eyerer, Near infrared (NIR) spectroscopy for in-line monitoring of polymer extrusion processes, Talanta. $\quad 50$ (1999) 283-290.

23. P.D. Coates, S.E. Barnes, M.G. Sibley, E.C. Brown, H.G.M. Edwards, I.J. Scowen, In-process vibrational spectroscopy and ultrasound measurements in polymer melt extrusion, Polymer. 44 (2003) 5937-5949.

24. A. Forster, J. Hempenstall, I. Tucker, T. Rades, The potential of small-scale fusion experiments and the Gordon-Taylor equation to predict the suitability of drug/polymer blends for melt extrusion, Drug Dev. Ind. Pharm. 27 (2001) 549-560.

25. D.J. Greenhalgh, A.C. Williams, P. Timmins, P. York, Solubility parameters as predictors of miscibility in solid dispersions, J. Pharm. Sci. 88 (1999) 1182-1190.

26. C. Heil, J. Hirsch, Improved process understanding and control of a hot-melt extrusion process with near-infrared spectroscopy, In: D. Douroumis (ed.) Hot-melt extrusion: pharmaceutical application, Oxford, John Wiley \& Sons Ltd. (2012) 333353.

27. A. Forster, J. Hempenstall, T. Rades, Characterization of glass solutions of poorly water soluble drugs produced by melt extrusion with hydrophilic amorphous polymers, J. Pharm. Pharmacol. 53 (3) (2000) 303-315.

28. R.J. Chokshi, H.K. Sandhu, R.M. Iyer, N.H. Shah, A.W. Malick, H. Zia, Characterization of physcio-mechanical properties of indomethacin and polymers to 
assess their suitability for hot-melt extrusion process as a means to manufacture solid dispersion/solution, J. Pharm. Sci. 94 (11) (2005) 2463-2474.

29. K. Izutsu, Y. Hiyama, C. Yomota, T. Kawanishi, Near-infrared analysis of hydrogenbonding in glass- and rubber-state amorphous saccharide solids, AAPS Pharm. Sci. Technol. 10 (2009) 524-529.

30. T. Hancewicz, A.d. Juan, M. Maeder, R. Tauler Ferré, Local rank analysis for exploratory spectroscopic image analysis. Fixed Size Image Window-Evolving Factor Analysis, Chemometr. Intell. Lab., 77 (1-2) (2005) 64-74.

31. M. Maeder, Evolving factor analysis for the resolution of overlapping chromatographic peaks, Anal. Chem, 59 (1987) 527-530.

32. M. Savolainen, A. Heinz, C. Strachan, K.C. Gordon, J. Yliruusi, T. Rades, N. Sandler, Screening for differences in the amorphous state of indomethacin using multivariate visualization, Eur. J Pharm. Sci., 30 (2007) 113-123.

33. C. Leuner, J. Dressman, Improving drug solubility for oral delivery using solid dispersions, Eur. J. Pharm. Biopharm. 50 (1) (2000) 47-60.

\section{List of Figures}

Fig. 1. The special die (A) made for in-line NIR monitoring via a transmission NIR probe and (B) an example of an extruded film.

Fig. 2. In-line NIR probes (transmission and reflectance) attached to the HME.

Fig. 1a. XRPD profiles of pure IND, SOL and VA64.

Fig. 3b. XRPD profiles of IND/SOL and IND/VA64 extrudates.

Fig. 4a. DSC thermograms of bulk IND, SOL and VA64.

Fig. 4b. DSC thermograms of physical mixtures of IND/VA64 and extruded formulations.

Fig. 4c. DSC thermograms of physical mixtures of IND/SOL and extruded formulations.

Fig. 5. Principal component score plot of NIR spectra from the in-line transmission probe data.

Fig. 6. NIR $2^{\text {nd }}$ derivative reflectance spectra of bulk IND, PM and in-line spectra of IND/VA64 (20/80, 40/60, w/w) extrudates collected from the first mixing zone (reflectance probe) and the die (transmission probe).

Fig. 6a. NIR $2^{\text {nd }}$ derivative reflectance spectra of bulk IND, PM and in-line spectra of IND/SOL (20/80, 40/60, 50/50, w/w) extrudates collected from the die (transmission probe). 
Fig. 7. Raman spectra of pure IND and VA64.

Fig. 8. a) Variation of eigenvalues with the principal components, b) comparison of first three PCs, c) comparison of first PC with the spectra of IND and VA64 and d) chemical map of PC1.

Fig. 9. Dissolution profiles for bulk IND, IND/SOL (50/50 wt/wt) and IND/VA64 (40/60 $\mathrm{wt} / \mathrm{wt})$.

\section{Appendix A. Supplementary material}

Fig. S1. XRPD profiles of physical mixtures of IND/SOL and IND/VA64.

Fig. S2. NIR $2^{\text {nd }}$ derivative reflectance spectra of PM and in-line spectra of IND/SOL (20/80, $40 / 60,50 / 50, \mathrm{w} / \mathrm{w}$ ) extrudates collected from the die (transmission probe) at $6500-7500 \mathrm{~cm}^{-1}$.

Fig. S3. NIR $2^{\text {nd }}$ derivative reflectance spectra of bulk IND, PM and in-line spectra of IND/SOL (20/80, 40/60, w/w) extrudates collected from the first mixing zone (reflectance probe) and the die (transmission probe) at $5850-6230 \mathrm{~cm}^{-1}$.

Fig. S4. Particle size distribution of micronized (cutter mill) IND/SOL (20/80, 40/60, 50/50 $\mathrm{wt} / \mathrm{wt}$ ) extruded sheets.

Fig.S5. Dissolution profiles for IND/SOL (20/80, 40/60, wt/wt) and IND/VA64 (20/80 $w t / w t)$. 